\title{
Effect of non-nutritive sucking and leg massage on physiological and behavioral indicators of pain following heel blood sampling in term neonates
}

\author{
Mehrdad Mirzarahimii ${ }^{1}$, Nasrin Mehrnoush ${ }^{* 2}$, Sahife Shahizadeh ${ }^{3}$, Nasrin Samadi $^{4}$, Firouz Amani $^{5}$ \\ ${ }^{I}$ Assistant Professor, Department of Pediatrics, Faculty of Ardabil University of Medical Sciences, Ardabil, Iran \\ ${ }^{2}$ MSC of Nursing, Ardabil University of Medical Sciences, Ardabil, Iran. \\ ${ }^{3}$ BSC of Nursing, Ardabil University of Medical Sciences, Ardabil, Iran \\ ${ }^{4}$ MSC of Nursing, Faculty of Nursing and Midwifery, Ardabil University of Medical Sciences, Ardabil, Iran \\ ${ }^{5}$ assistant professor of Biostatistics, Faculty of medicine, Ardabil University of Medical Sciences, Ardabil, Iran \\ *Corresponding author E-mail: n.mehrnoush@arums.ac.ir
}

\begin{abstract}
Background: Pain management is especially important for neonates who are not able to verbally express their pain. Non-pharmacological pain management method as an alternative to pharmacological pain medication has increased nowadays.

Objectives: The purpose of this study was to test the effect of leg massage and non-nutritive sucking (NNS) on reducing the pain that neonates experience when undergoing the heel stick procedure for blood testing.

Methods: This investigation was a random controlled clinical trial study on ninety neonates who were selected randomly among admitted neonates to NICU in Alavi hospital at Ardabil. The subjects were randomly classified in two case and one control groups. The neonates received leg massage $(n=30)$, NNS $(n=30)$, and no intervention ( $n=30)$ respectively. Measurements of $\mathrm{HR}$ and $\mathrm{SaO} 2$ were taken twice: once before and then again after intervention. Pain response was measured by the PIPP scale.

Results: The study showed that the HR of the three groups significantly increased after heel stick compared to before heel stick. The change of $\mathrm{SaO} 2$ levels in NNS groups was lower than the control and massage groups. The change of PIPP scores in massage and NNS groups was lower than that of the control group; however, there was not statistically significant differences between NNS \& massage groups $(\mathrm{p}=0.91)$

Conclusion: pain management and pain relief in the infant is an important issue in neonatal health care. It is suggested that the single or combined use of massage and a pacifier is effective in reducing pain responses in neonates undergoing heel stick.
\end{abstract}

\section{Background}

Pain management is especially important for neonates who are not able to verbally express their pain. The traditional view that neonates are not capable of perceiving pain has been refuted and there is now no doubt those neonates perceive pain [1]. Although infants do not verbalize, they reveal their vulnerability to pain through specific pain behaviors and physiologic changes [2]. The physiological indicators of pain include autonomic changes in the heart and respiratory rate, blood pressure, and oxygen saturation. There are also hormonal responses to pain, but these need a laboratory evaluation to make an informed judgment. Behavioral responses consist of Changes in sleeping/waking patterns, crying, body and limb movement, and facial expressions [3]. Pain can be managed by pharmacological and non pharmacological interventions. Using analgesics to relieve short-term procedural pain in newborns is questionable because of these agents' poor effectiveness and potential side effects [4]. To avoid adverse effects of pharmacologic analgesic agents, non pharmacologic strategies to minimize neonatal procedural pain, such as skin-to-skin contact with a caregiver, non-nutritive sucking (NNS), oral administration of sweeteners, and massage have been proposed [5].

Non-nutritive sucking is a comfort measure for infants and helps to make them calm [6]. NNS is hypothesized to produce analgesia by stimulating orotactile and mechanoreceptors in the mouth, thus modulating transmission or processing of nociception by the endogenous non opioid system [2]. Massage therapy is most popularly used with pain 
syndromes[7] Gentle massage may inhibit the transmission of pain along the ascending fibres by closing the gate or by activating the descending endogenous opioid and non-opioid pathways to decrease nociceptive transmission and reduce pain [2]. Pain stimuli in neonates generate short- and long-term effects. Short-term effects include physiologic responses (increase in heart and breath rate, decrease in oxygen saturation, and increase in intracranial pressure) and behavioral responses (brow bulge, eye squeeze, nasolabial furrow, and cry) Although long-term effects are less easy to prove, some studies on circumcised boys showed that newborn early pain experience can alter pain response in later infancy [8].Measuring pain in newborns is a complex problem. There has been extensive use of different physiologic and behavioral indicators. Current knowledge speaks in favor of using composite scales including both physiologic and behavioral measures. One of the most frequently applied methods is the Premature Infant Pain Profile (PIPP). The PIPP was developed to assess acute pain in preterm and full-term newborns. Other signs of distress, such as crying and heart rate, are also used as pain indicators [9]

The purpose of this study was to examine an effective pain management method for neonates during heel stick. It compared the effects of massage, non-nutritive sucking and usual care on full-term newborn infants who experienced heel stick with each other. The instruments used in the present study were the Premature Infant Pain Profile (PIPP), heart rate (HR) and oxygen saturation level $(\mathrm{SaO} 2)$.

\section{Materials and methods}

\subsection{Samples}

Ninety neonates (i.e. leg massage group, $n=30$; NNS group, $n=30$; control group, $n=30$ ) participated in the study. Criteria for inclusion were: full term of the neonate (i.e. over 37 weeks of gestation), 1-7 day newborns were admitted to the NICU, physiologically stable and without neurological abnormalities or anomalies. The neonates under sedation or with neurological or congenital anomalies were excluded from the study due to any possible alteration/difference in their behavioral or pain response to the procedural stimulation.

\subsection{Procedure}

This was a random controlled clinical trial study. Ninety neonates were selected randomly from admitted neonates to NICU in Alavi hospital at Ardebil. The neonates were recruited by a researcher who was a nurse. She obtained written informed consent from all the parents. Then, the subjects were randomly classified into two case and one control groups. Ninety neonates were recruited, 30 cases were assigned to each group. The neonates from the first group received leg massage, those from the second group received NNS and those from the last group received no intervention.The assistant nurse performed the heel stick using a lancet. All the blood sampling in the study was performed for clinical purposes, such as newborn screening tests. Standard protocol for blood sampling involved swabbing the heel with a small gauze pad with disinfectant, lancing the heel and then gently squeezing the heel intermittently until the amount of blood required for clinical care was collected.

In this study, interventions (i.e. leg massage and NNS) were provided for $2 \mathrm{~min}$ prior to starting the heel stick procedure and lasted until the completion of the procedure. The assistant nurse chose the heel for blood collection. Two minutes before the heel stick, one of the investigators slowly massaged the outer aspect of the leg chosen for the heel stick from toes to mid thigh by using a firm but gentle pressure by fingers and thumbs. The massage pressure was adjusted to keep the baby comfortable. At the end of the massage intervention $(2 \mathrm{~min})$, the heel was wrapped with a warm cloth for 1 min. An assistant nurse performed the heel stick using a lancet. The blood sample was collected for newborn screening thyroid tests. During the NNS intervention, the researcher gave each neonate a standard, small, short, hollow soft latex nipple while applying gentle pressure to maintain it in the neonate's mouth. Control group neonates received no intervention during the procedure. Measurements of $\mathrm{HR}$ and $\mathrm{SaO} 2$ were taken twice: once before providing intervention (i.e. pre-test) and then again after completion of the intervention (i.e. post-test). Pain response was measured by the PIPP. The PIPP scale is a validated 7-indicator scale for the assessment of acute pain in preterm and term infants. It measures gestational age, behavioral state, heart rate, oxygen saturation, and 3 facial actions (brow bulge, eye squeeze, and nasolabial furrow). Score ranges from 0 (no pain) to 21(maximum pain). Because the first item was gestational age, and in our study the eligibility criteria included only term neonates, thus the maximum rate on the PIPP scale was 18 [9].

\subsection{Data analysis}

All data were managed with confidentiality and analyzed using SPSS 14.0. Parametric statistics were used for data analysis after testing for the normal distribution of the data. All values were expressed as means and SDs. Comparisons 
were made within each group using paired t-tests and between groups using analysis of variance (ANOVA). A twotailed P-value less than 0.05 was considered to be significant.

\section{Results}

As shown in Table 1, there were no significant differences among the infants in the three groups based on the demographic variables including sex, age, birth weight and delivery using Chi-square. Overall, neonates weighed between 3.0 and $3.5 \mathrm{~kg}$, and their age was 3-4days (table 1).

\begin{tabular}{|c|c|c|c|c|c|c|c|}
\hline \multirow[b]{2}{*}{ Characteristics } & \multicolumn{2}{|c|}{$\begin{array}{l}\text { Leg massage } \\
N=30\end{array}$} & \multicolumn{2}{|c|}{$\begin{array}{l}N N S \\
N=30\end{array}$} & \multicolumn{2}{|c|}{$\begin{array}{l}\text { Control } \\
N=30\end{array}$} & \multirow[t]{2}{*}{ P value } \\
\hline & $\mathrm{N}$ & $\%$ & $\mathrm{~N}$ & $\%$ & $\mathrm{~N}$ & $\%$ & \\
\hline Age & & & & & & & $P=0.68$ \\
\hline $3-4$ & 26 & 86.7 & 27 & 90 & 25 & 83.3 & \\
\hline $5-6$ & 4 & 13.3 & 3 & 10 & 4 & 13.3 & \\
\hline$>6$ & 0 & 0 & 0 & 0 & 1 & 3.3 & \\
\hline Sex & & & & & & & $\mathrm{P}=0.87$ \\
\hline Male & 19 & 63.3 & 17 & 56.7 & 18 & 60 & \\
\hline Female & 11 & 36.7 & 13 & 43.3 & 12 & 40 & \\
\hline Weight & & & & & & & $P=0.99$ \\
\hline $2000-2500$ & 2 & 6.7 & 2 & 6.7 & 2 & 6.7 & \\
\hline $2501-3000$ & 9 & 30 & 8 & 26.7 & 9 & 30 & \\
\hline $3001-3500$ & 11 & 36.7 & 14 & 46.7 & 12 & 40 & \\
\hline$>3500$ & 8 & 26.7 & 6 & 20 & 7 & 23.3 & \\
\hline Delivery & & & & & & & $\mathrm{P}=0.15$ \\
\hline NVD & 8 & 26.7 & 15 & 50 & 10 & 33.3 & \\
\hline $\mathrm{C} / \mathrm{S}$ & 22 & 73.3 & 15 & 50 & 20 & 66.7 & \\
\hline
\end{tabular}

The HR of the three groups significantly increased after heel stick compared to the time before heel stick. The SaO2 levels of all three groups of neonates decreased after heel stick compared to before heel stick (Table 2).

\begin{tabular}{lllllc}
\multicolumn{2}{c}{ Table 2: Comparison of heart rate and oxygen saturation among within groups (N=90) } \\
\hline Group & Pretest & $\begin{array}{l}\text { Post test } \\
\text { M(SD) }\end{array}$ & Difference & \multicolumn{2}{c}{ Within a group } \\
HR & M(SD) & & & T & p \\
NNS & $136.46(16.93)$ & $151.70(12.11)$ & $-15.23(10.85)$ & -7.68 & 0.000 \\
Massage & $127.00(12.80)$ & $142.13(12.11)$ & $-15.13(10.68)$ & -7.75 & 0.000 \\
Control & $133.20(13.25)$ & $148.60(11.91)$ & $-15.40(8.66)$ & -9.73 & 0.001 \\
Sao2 & & & & & \\
NNS & $94.70(2.65)$ & $92.73(2.85)$ & $1.96(1.82)$ & 5.89 & 0.000 \\
Massage & $94.13(2.35)$ & $91.10(2.68)$ & $3.03(2.04)$ & 8.13 & 0.000 \\
Control & $93.90(2.84)$ & $89.86(3.55)$ & $4.03(3.60)$ & 6.12 & 0.001 \\
\hline
\end{tabular}

The change of $\mathrm{SaO} 2$ levels in NNS groups was lowers than that of the control and massage groups. There was a significant difference in the change of $\mathrm{SaO} 2$ levels between intervention groups (i.e. massage or NNS group) and the control group. No difference was found between the massage and NNS groups. But there was a significant difference in the change of $\mathrm{SaO} 2$ levels between NNS and control groups $(\mathrm{p}=0.001)$ (Table 3$)$.

The pain scores (PIPP) of the neonates in the three groups were shown to have significant difference after heel stick. The change of PIPP scores in massage and NNS groups was lower than that of the control group; however, there was not statistically significant differences between NNS \& massage groups(p=0.91) (Figure 1). 
Table 3: Comparison of pain score, oxygen saturation and heart rate among groups $(\mathrm{N}=90)$

\begin{tabular}{llll}
\hline Groups & comparison & Between group & $\mathrm{p}$ \\
\hline HR & & $\mathrm{t}$ & 0.10 \\
NNS & Mag vs. Ctr & -6.46 & 0.058 \\
Massage & NNS vs. Ctr & 3.10 & 0.91 \\
Control & Mag vs. NNS & $0 / 30$ & 0.26 \\
Sao2 & & & 0.001 \\
NNS & Mag vs. Ctr & 1.23 & 0.10 \\
Massage & NNS vs. Ctr & 2.86 & 0.02 \\
Control & Mag vs. NNS & -1.63 & 0.009 \\
PIPP & & & 0.91 \\
NNS & Mag vs. Ctr & -1.90 & -2.20 \\
Massage & NNS vs. Ctr & 0.30 & \\
Control & Mag vs. NNS & & \\
\hline
\end{tabular}

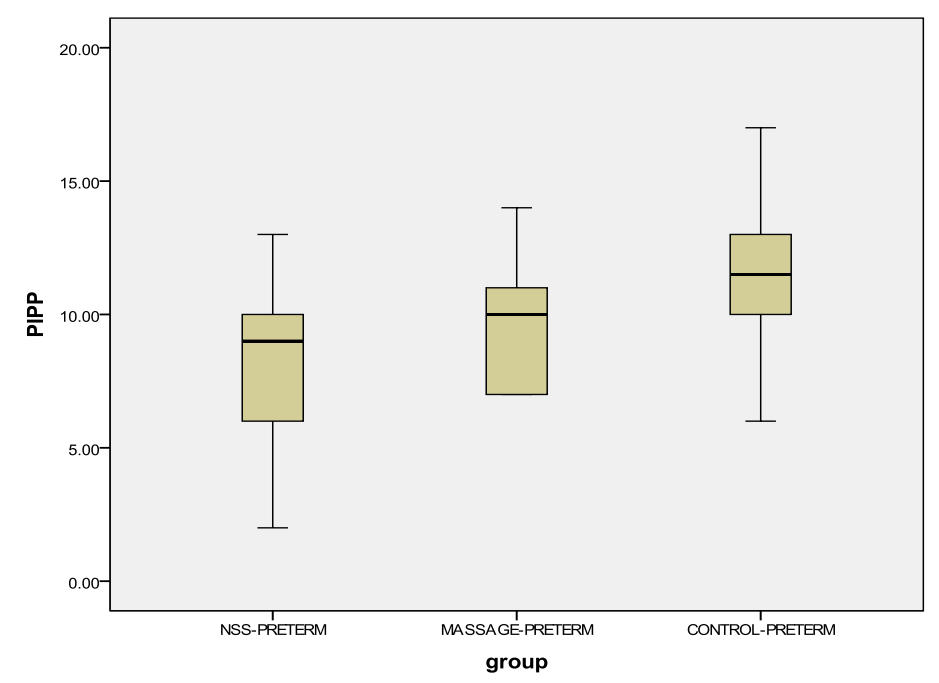

Fig1: Comparison of pain (PIPP) score among groups $(\mathrm{N}=90)$

\section{Discussion}

A number of non-pharmacologic therapies have been shown to be beneficial to the management of mild to moderate pain in the neonate. [10] These therapies include nonnutritive sucking both with and without sucrose, swaddling or kangaroo care, music therapy, and multi-sensorial stimulation. Nonnutritive sucking has been shown to significantly reduce crying and pain responses in neonates, in response to heel lance procedures and circumcision. [11] The physiological mechanisms for this response remain unclear, but may be related to stimulating serotonin release in the brainstem. The addition of sucrose to the pacifier adds analgesic potency and appears to be mediated by an endorphin effect. [12]

In our study, The $\mathrm{SaO} 2$ levels of all three groups of neonates decreased after heel stick, compared to before heel stick. When the $\mathrm{SaO} 2$ levels measured before and after heel stick were compared, the decrease in $\mathrm{SaO} 2$ level was shown to be insignificant only in the NNS group neonates. Also all three groups showed increased HR when they were measured 2 min after heel stick. This result can be understood to mean that neither Massage nor NNS could help the neonates maintain their HR throughout heel stick. The difference between NNS \& control groups can suggest that NNS in comparison to control lead to less reduction of Sao2 than massage and can be applied instead of other techniques. It suggests that NNS method could be considered another alleviating intervention for neonatal pain. For newborns younger than one day, NNS is the best choice to relieve pain [4]. One meta-analysis confirmed that the type of pacifier (shape, material) and the duration of sucking can influence the physiological pain indicators [13].In another similar study, infants with pacifier demonstrated significantly lower scores on the PIPP scale than control group only during heel-lance and, interestingly, there was no statistically significant difference between the pacifier group and other 
groups receiving pacifier and sucrose [14]. South et al in the study showed that NNS significantly decreased some elements of measurable physiologic pain response of the neonate during circumcision [15].Phillips et al studied 3 groups of newborns undergoing blood collection in respect of crying behavior: breastfeeding, using pacifier held by mothers, and using pacifier held by an assistant. They found that breastfeeding and maternal holding reduce crying behavior during heel stick procedures [16]. These findings support our hypothesis that NNS have soothing effects on pain.

Massage was the second intervention on neonates in our study. Gentle massage may inhibit the transmission of pain along the ascending fibers by closing the gate or by activating the descending endogenous opioid and non-opioid pathways to decrease nociceptive transmission and reduce pain [2]. It may also be that massage which contributes to a soothing environment similar to studies of multisensory stimulation potentiating the analgesic effects of oral sugar in newborns undergoing heel sticks [17]. The HR of the three groups significantly increased after heel stick compared to before heel stick. The SaO2 levels of all three groups of neonates decreased after heel stick compared to before heel stick. Two interventions (massage and NNS) made HR increase after intervention in comparison with before, and there were statistically significant differences between groups of interventions and control in terms of feeling pain. These effects can be attributed to these confounding factors. Some authors have found a decrease in SpO2 in response to procedural pain. They saw a small $(1.8 \%)$ decrease in $\mathrm{SpO} 2$ with no prior massage [2].

These findings support our hypothesis that massage has soothing effects on pain. One of the goals of pain management is to maintain behavioral stability. For this purpose, we assessed it with PIPP scale. The pain scores (PIPP) of the neonates in the three groups revealed significant difference after heel stick. The change of PIPP scores in massage and NNS groups was lower than that of the control group. It means that interventions had positive effect on case groups, but both groups (NNS\& Massage) exceeded control group in a similar way. This result showed that NNS \& Massage can be used interchangeably. Some studies used the composite Premature Infant Pain Profile's (PIPP) score as the outcome measure showed significant reduction in PIPP with sucrose at 30 and $60 \mathrm{~s}$ after heel-lance[18].Gibbons \& Stevens found that sucrose solution followed by non-nutritive sucking was the most effective intervention in reducing PIPP scores during punctures for three groups of neonates with gestational ages of 27 to 31 weeks, 32 to 35 weeks and 36 to 42 weeks, when compared with sucrose alone or sterile water followed by non-nutritive [19]. Our findings in the present study are consistent with Gibbons \& Stevens's finding and confirm our hypothesis.

There are two limitations in this study which should be considered in further studies. One is that research nurses who performed PIPP measuring were not blinded to the treatment group, which could bring a potential bias. The other is that Infant facial responses to pain as well as physiological responses might have been influenced by multiple confounding factors, such as infant hunger or discomfort, temperament, sleep/wake state, and prior painful experiences. Although some of these variables were controlled in our analyses, future studies should consider these factors in selecting newborns and in data analysis. Despite these limitations, this study introduced two interventions that may be an effective pain management intervention in infants. Therefore, this study could provide health professionals with a research-based intervention.

\section{Conclusions}

Some painful, invasive procedures, such as heel sticks, are commonly performed in neonates, as they are necessary for care. Neonates are as sensitive to painful stimuli as adults are and pain experienced in the neonatal period might have long-term effects later in life. Therefore, pain management and pain relief in the infant is an important issue in neonatal health care. We suggest that the single or combined use of massage and a pacifier is effective in reducing pain responses in neonates undergoing heel stick and the use of these simple pain relieving interventions should be considered.

\section{Acknowledgements}

This study was supported by deputy for Research, Ardabil University of Medical Sciences. The authors also wish to thank NICU nursing staff of Alavi Hospital for their great help during this study.

\section{References}

[1] H. Im, E. Kim, E. Park, K. Sung, W. Oh, Pain Reduction of Heel Stick in Neonates: Yakson Compared to Non-nutritive Sucking, Journal of Tropical Pediatrics 54(2007) 31-35.

[2] S.Jain, P.Kumar,D. D. McMillan, Prior leg massage decreases pain responses to heel stick in preterm babies, Journal of Paediatrics and Child Health 42 (2006) 505-508. 
[3] M. Eriksson, H.Storm, A .Fremming, J. Schollin, Skin conductance compared to a combined behavioural and physiological pain measure in newborn infants, Acta Pædiatrica97( 2008) 27-30.

[4] J. J. Liaw, W.P. Zeng, L. Yang, Y. S. Yuh, T. Yin, M.H. Yang, Nonnutritive Sucking and Oral Sucrose Relieve Neonatal Pain During Intramuscular Injection of Hepatitis Vaccine, Journal of Pain and Symptom Management 42 (2011)42-48.

[5] A. G. Chermont, L. F. M. Falcão, E. H. L. de Souza Silva, R. C. X. Balda, R. Guinsburg, Skin-to-Skin Contact and/or Oral 25\% Dextrose for Procedural Pain Relief for Term Newborn Infants, Pediatrics 124(2009) e1101.

[6] L.A. Hatfield, Sucrose decreases infant biobehavioral pain response to immunizations: A randomized controlled trial, Journal of Nursing Scholarship 40 (2008) 219-225.

[7] T. Field, M. Diego, M. Hernandez-Reif, Massage therapy research, Developmental Review 27 (2007) 75-89.

[8] L. Codipietro, M. Ceccarelli, A. Ponzone, Breastfeeding or Oral Sucrose Solution in Term Neonates Receiving Heel Lance: A Randomized, Controlled Trial, Pediatrics 122( 2008) e716-e721.

[9] R.B. Jonsdottir, G. Kristjansdottir, The sensitivity of the premature infant pain profile: PIPP to measure pain in hospitalized neonates, J Eval Clin Pract,11( 2005) 598-605.

[10] J.E. Jones, N. Kassity, Varieties of alternative experience: complementary care in the neonatal intensive care unit, Clin Obstet Gynecol 44(2001) 750- 768 .

[11] M.M.T. South, R.A. Strauss, A.P. South, et al, The use of non-nutritive sucking to decrease the physiologic pain response during neonatal circumcision: A randomized controlled trial, Obstet Gynecol 193(2005)537- 543.

[12] G.R. Kracke, K.A. Uthoff, J.D. Tobias: Sugar solution analgesia: the effect of glucose on expressed Mu opioid receptors. Anesth Analg 101(2005)64-68.

[13] E. Cignacco, J. P.H. Hamers, L. Stoffel, et al, The efficacy of non-pharmacological interventions in the management of procedural pain in preterm and term neonates. A systematic literature review, European Journal of Pain 11 (2007) 139-152.

[14] A. Leslie, N. Marlow, Non-pharmacological pain relief, Seminars in Fetal \& Neonatal Medicine 11(2006) $246-250$.

[15] M. M. T. South, R. A. Strauss, A. P. South, J. F. Boggess, J. M. Thorp, The use of non-nutritive sucking to decrease the physiologic pain response during neonatal circumcision: A randomized controlled trial, American Journal of Obstetrics and Gynecology 193 (2005) $537-43$.

[16] R.M. Phillips, C.J. Chantry, M.P. Gallagher, Analgesic effects of breast-feeding or pacifier use with maternal holding in term infants. Ambul Pediatr. 5(2005) 359-364.

[17] C.V. Bellieni, F. Bagnoli, S. Perrone et al, Effect of multisensory stimulation on analgesia in term neonates: a randomized controlled trial, Pediatr. Res. 51(2002) 460-3.

[18] S. Gibbins, B.Stevens , E. Hodnett, J. Pinelli, A. Ohlsson,G. Darlington, Efficacy and safety of sucrose for procedural pain relief in preterm and term neonates. Nurs Res 51(2002) 375-82.

[19] S. Gibbins,B. Stevens, The influence of gestational age on the efficacy and short-term safety of sucrose for procedural pain relief. Adv Neonatal Care 3(2003)241-9. 